\title{
Operational status of the Magnum-PSI linear plasma device
}

\author{
John Scholten*, P.A. Zeijlmans van Emmichoven, H.J.N. van Eck, P.H.M. Smeets, \\ G.C. De Temmerman, S. Brons, M.A. van den Berg, H.J. van der Meiden, M.J. van de Pol, \\ M.F. Graswinckel, P.W.C. Groen, A.J. Poelman, J.W. Genuit
}

FOM Institute DIFFER - Dutch Institute for Fundamental Energy Research, Association EURATOM-FOM, Trilateral Euregio Cluster, PO Box 1207, 3430BE Nieuwegein, The Netherlands

\section{H I G H L I G H T S}

- High heat flux, high density plasmas in a highly accessible linear plasma device.

- Plasma exposure of targets of different sizes under selectable plasma beam angles.

- Dedicated plasma and surface diagnostics.

- Differential vacuum pumping system.

\section{A R T I C L E I N F O}

\section{Article history:}

Received 14 September 2012

Received in revised form 15 May 2013

Accepted 16 May 2013

Available online 17 June 2013

\section{Keywords:}

ITER

Magnum-PSI

Plasma generator

Plasma surface interactions

\begin{abstract}
A B S T R A C T
The construction phase of the linear plasma generator Magnum-PSI at the FOM institute DIFFER has been completed and the facility has been officially opened in March 2012. The scientific program to gain more insight in the plasma-wall interactions relevant for ITER and future fusion reactors has started.

In Magnum-PSI, targets of a wide range of materials and shapes can be exposed to high particle, high heat flux plasmas $\left(>10^{24}\right.$ ions $\left.\mathrm{m}^{-2} \mathrm{~s}^{-1} ;>10 \mathrm{MW} / \mathrm{m}^{2}\right)$. For magnetization of the plasma, oil-cooled electromagnets are temporarily installed to enable pulsed operation until the device is upgraded with a superconducting magnet. The magnets generate a field of up to $1.9 \mathrm{~T}$ close to the plasma source for a duration of $6 \mathrm{~s}$. Longer exposure times are available for lower field settings.

Plasma characterizations were done with a variety of gases ( $\mathrm{H}, \mathrm{D}, \mathrm{He}, \mathrm{Ne}$ and $\mathrm{Ar}$ ) to determine the machine performance and prepare for subsequent scientific experiments. Thomson scattering and optical emission spectroscopy were used to determine the plasma parameters while infrared thermography and target calorimetry were used to determine the power loads to the surface.

This paper reports on the status of Magnum-PSI and its diagnostic systems. In addition, an overview of the plasma parameters that can be achieved in the present state will be given.
\end{abstract}

(c) 2013 Elsevier B.V. All rights reserved.

\section{Introduction}

Magnum-PSI is a linear plasma device dedicated to the study of plasma-wall interactions under fusion relevant conditions, and is designed around a $3 \mathrm{~T}$ superconducting magnetic field [1]. Due to the acceptance test failures of the superconducting magnet, a temporary solution with conventional coils has been realized for the creation of pulsed magnetized plasmas. This led to the first magnetized plasma in 2011 and subsequently a commissioning period during which extensive plasma characterizations were performed to determine the machine performances in this configuration. Different gas types and flows, magnetic fields, discharge currents and

\footnotetext{
* Corresponding author.

E-mail address: j.scholten@differ.nl (J. Scholten).
}

target bias voltages were used. Plasma density and temperature were determined by Thomson scattering, surface temperatures by infrared imaging while the total power deposited on the target was determined by calorimetry.

\section{Magnum-PSI installation}

Magnum-PSI is a $\sim 15 \mathrm{~m}$ linear plasma device, consisting of a water cooled vacuum vessel with a cascaded arc plasma source mounted on a manipulator tube at one end and a target holder manipulator tube at the other end. Both can be brought within $1 \mathrm{~m}$ distance from each other with servo motor controllers. A magnetic field at this position allows the generation of a plasma beam between source and target. For plasma- and target-diagnostics, the vessel at the target location is fitted with many ports and windows of several sizes (Fig. 1). Large roots pumps $\left(30.000 \mathrm{~m}^{3} / \mathrm{h}\right)$ are 


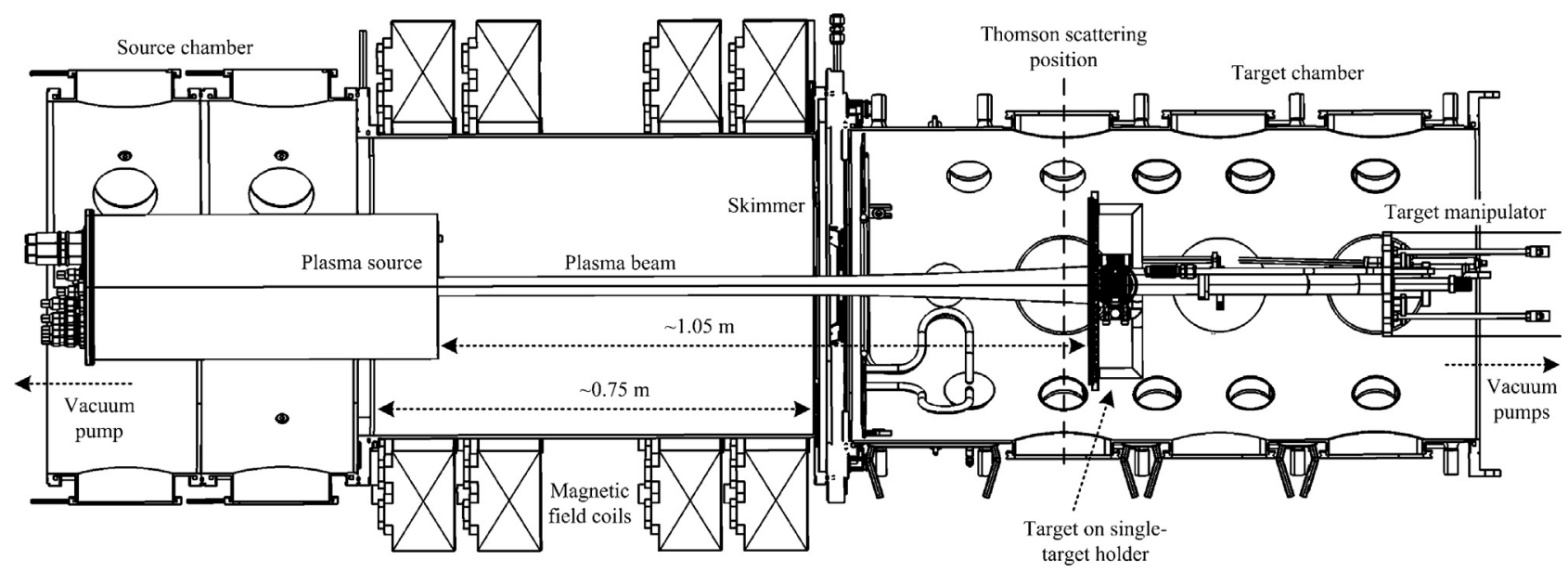

Fig. 1. Schematic drawing of Magnum-PSI with the temporary magnet coils between source and target.

connected to both ends of the vacuum vessel to allow a low vacuum pressure ( $\sim 1$ Pa or lower at the target) during plasma operation. In addition, connected turbo pumps enable a very low pressure $\left(<10^{-4} \mathrm{~Pa}\right)$ in between operations to keep the contamination of the vessel to a minimum.

The target manipulator allows remote control of the target rotation and tilting for exposures under an angle and for diagnostic convenience. Two target holder types are available: a single-target holder for large targets and a multi-target holder for up to 5 smaller targets. Both are water-cooled with flow up to $50 \mathrm{l}$ per min. The heat conductance between target and holder can be varied by the shape and thickness of the material (typically Grafoil) in between. The target holder can be retracted to the target exchange and analysis chamber (TEAC) with its separate vacuum pump system [2]. Power supplies can be connected to the target, via the manipulator, to vary the target voltage during plasma exposure. In the retracted position, a beam dump is moved in front of the plasma source to prevent target exposure when tuning the plasma source to the preferred plasma conditions.

On the source manipulator water cooled cascaded arc sources [3] of different configurations can be installed depending on the requirements for plasma density and plasma temperature ranges. During plasma operation, the gas flows (up to $66.7 \mathrm{~Pa} \mathrm{~m}^{3} / \mathrm{s}$ per gas), gas types and cathode to anode current can be changed. Six power supply units ( $300 \mathrm{~V}, 100 \mathrm{~A}$ each) are available for the plasma source current and target bias voltage. The units can supply a $700 \mathrm{~V}$ pulse for the ignition of the plasma. In addition, a capacitor bank has been added recently to the installation to allow repetitive high power loads on the target by shortly increasing the cathode current up to several $\mathrm{kA}[4]$.

The complete installation, including the diagnostics, can be controlled form the central control room with direct view onto Magnum-PSI. As part of the CODAC (Control, Data Acquisition and Communication) system, a central database is in operation. In addition a central GPS-time synchronized timing system with a 1 us resolution is in operation for triggering diagnostic systems and recording the corresponding timestamps. A fast general purpose data acquisition system integrated with the CODAC system is under development. Tools for viewing and exporting the data stored in the database are available. Database libraries are currently only available for python. More details on the CODAC software architecture is presented at this conference as well [5].

\subsection{Temporary magnet}

Until a superconducting magnet is available, pulsed mode operation is possible with the integration of a magnet consisting of 4
Table 1

Magnetic field and pulse duration options.

\begin{tabular}{lclllc}
\hline Setting & $I_{\text {COILS }}(\mathrm{kA})$ & $B_{\text {MAX }}(\mathrm{T})$ & $B_{\text {SOURCE }}(\mathrm{T})$ & $B_{\text {TARGET }}(\mathrm{T})$ & Time $(\mathrm{s})$ \\
\hline 1 & 4.3 & 0.47 & 0.43 & 0.08 & 112 \\
2 & 8.7 & 0.95 & 0.87 & 0.16 & 27 \\
3 & 13.0 & 1.41 & 1.30 & 0.25 & 12 \\
4 & 17.3 & 1.88 & 1.73 & 0.33 & 6 \\
\hline
\end{tabular}

oil-cooled bitter coils. This required changes to the vacuum vessel. Of the 2 skimmers for blocking neutral gas and thus lowering the pressure at the target, one skimmer has been removed. In addition the roots pump system previously connected to the section between the 2 skimmers cannot be used due to the smaller bore of the magnetic field coils. The vacuum vessel at the location of the coils has been replaced by a small diameter vessel. The pressure at the target is however well below the requirement $(<3 \mathrm{~Pa})$ with the currently used gas flows at the source (ranging from 4-20 $\mathrm{Pa} \mathrm{m}^{3} / \mathrm{s}$ or 2.4-10 standard liter per minute). The highest pressure in the target chamber, during normal operation, was $0.85 \mathrm{~Pa}$ with $16.7 \mathrm{~Pa} \mathrm{~m}^{3} / \mathrm{s}$ $\mathrm{H}_{2}$ gas inlet, $200 \mathrm{~A}$ discharge current and highest magnetic field setting. The pressure in the source chamber was 2.25 Pa during this discharge.

The target chamber, in which the target is located during plasma exposure, has not been changed because of the impact on the diagnostic systems such as Thomson scattering. As a result, the target cannot be placed inside the magnet and has as a consequence a lower field than at the plasma source. The target is thus exposed in an expanding magnetic field region, which increases the plasma beam diameter. Table 1 shows the magnetic field at the positions of the target and the source as well as the maximum pulse length for the four possible current settings. Due to heating up of the coils, the current, and therewith the magnetic field, decreases 10-20\% (depending on the setting) at the end of the maximum pulse time.

\section{Plasma properties}

The plasma properties (such as electron density and temperature) close to the target, depend on many factors, amongst which: the type of plasma source, the gas inlet type and flow at the source, the plasma source cathode current, the magnetic field strength and the position and inclination of the target.

Fig. 2 shows an overview of the achieved peak electron temperature and density as measured by Thomson scattering in the current Magnum-PSI configuration. The highest temperatures were obtained with a 3-cathode 'trumpet' source with full copper plasma channel plates at magnet setting 2 . In this source the plasma 


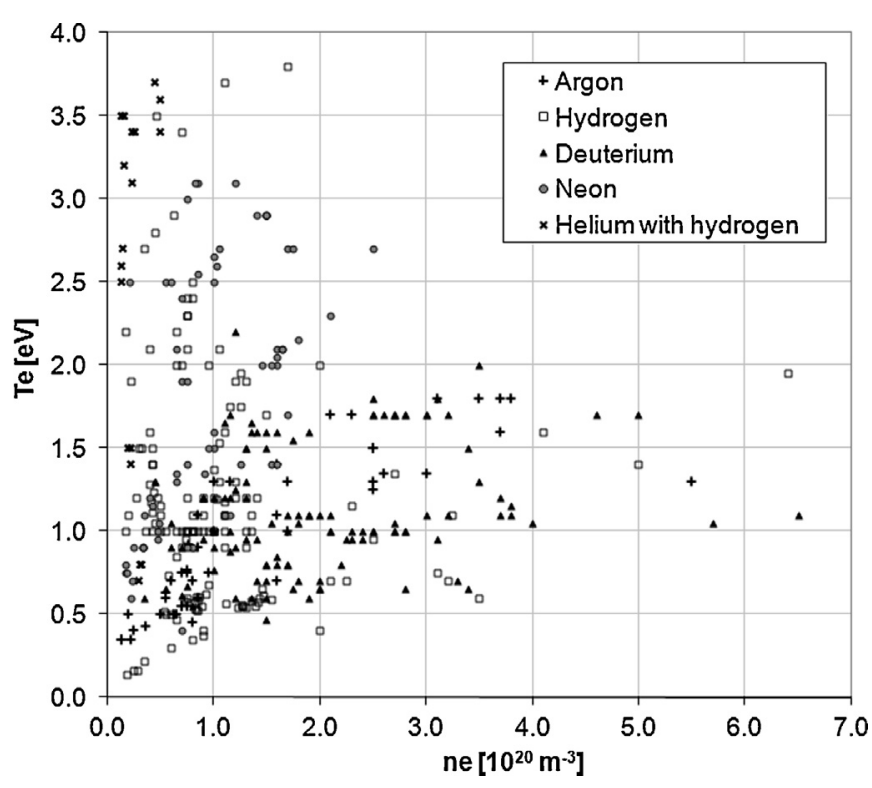

Fig. 2. Thomson scattering results from various experiments performed. Center electron density $\left(n_{\mathrm{e}}\right)$ and center electron temperature $\left(T_{\mathrm{e}}\right)$.

channel diameter increases from cathode to nozzle. Currently, this type of source with molybdenum inserts and only 1 cathode is being tested.

The highest densities were created with a $7 \mathrm{~mm}$ straight channel source (also with molybdenum inserts) and high cathode currents (250 A) at magnet setting 3.

The current set up of the magnetic coils causes the magnetic field lines to diverge in the direction of the target by a factor of 2.5. This divergence results in a broadening of the plasma beam diameter and a decrease in the plasma density. Fig. 3 shows a plasma profile measured by Thomson scattering $27 \mathrm{~mm}$ in front of the target position. The corresponding plasma was created with a single cathode, $7 \mathrm{~mm}$ channel source using $8 \mathrm{~Pa} \mathrm{~m}^{3} / \mathrm{s}$ hydrogen flow, 250 A cathode current and the magnet at setting 3 .

The total power load on the target can be determined with calorimetry using water flow and temperature sensors. The system is calibrated with an electrical heater in series with the target. Fig. 4 shows the power load as function of cathode current for different

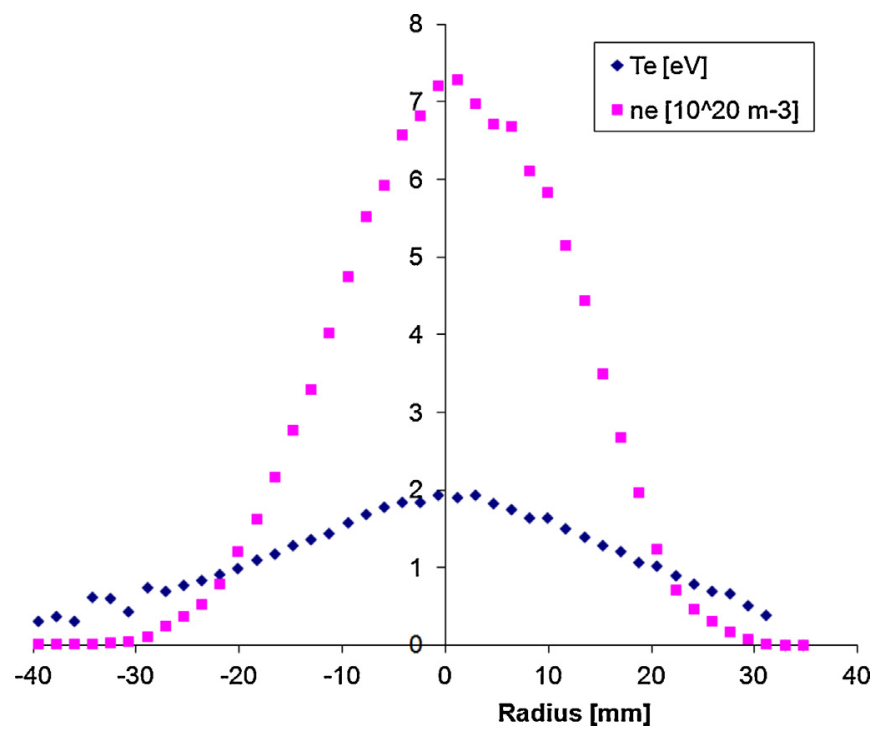

Fig. 3. Thomson profiles of a hydrogen plasma.

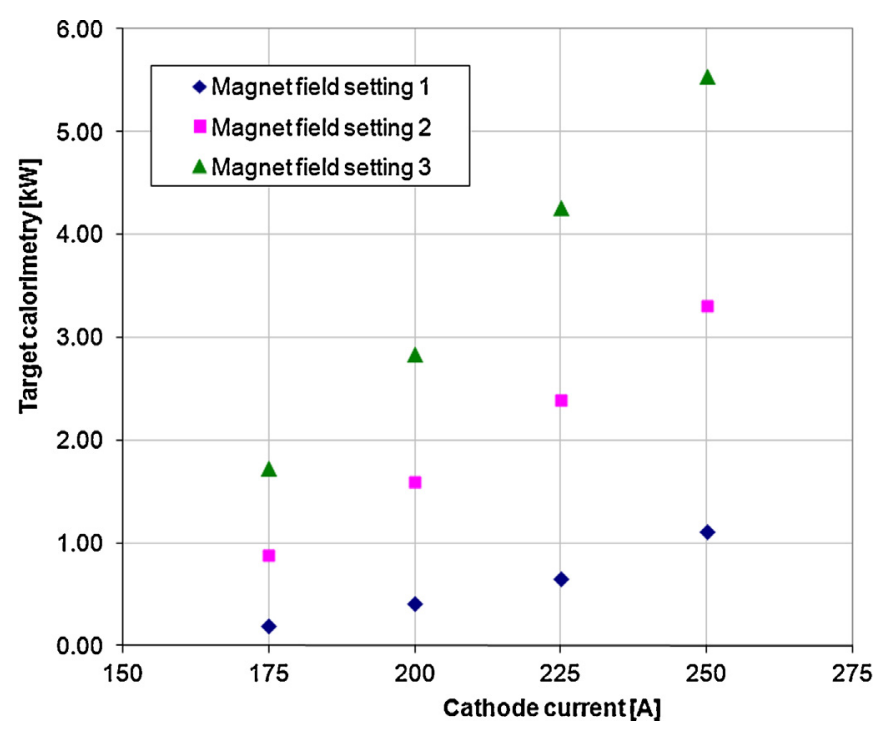

Fig. 4. Target calorimetry versus cathode current for 3 magnetic field settings using a $7 \mathrm{~mm}$ channel plasma source, $8 \mathrm{~Pa} \mathrm{~m}^{3} / \mathrm{s}$ hydrogen inlet flow.

magnetic field strengths. The top-right data point (250 A, magnet setting 3 ) is the same pulse in which with the Thomson scattering data in Fig. 3 was measured.

Assuming a (2D-) Gaussian distribution profile of the power and the density of the plasma beam, then half of the total power is contained by the beam inside the FWHM area, which $0.5 \times 5.54 \mathrm{~kW}$ in $6.2 \times 10^{-4} \mathrm{~m}^{2}$ for the plasma corresponding to Fig. 3, then the average power density is $\sim 4.5 \mathrm{MW} / \mathrm{m}^{2}$ in this area. The actual values are likely higher because the temperature in the center is higher. At the center of the beam the power density will be close to $10 \mathrm{MW} / \mathrm{m}^{2}$. This is in the range of the design goal for Magnum-PSI.

Calculating the particle flux using Thomson scattering data requires the knowledge of the axial ion velocity. This data is not yet available for Magnum-PSI. A rough estimation can be done by using the pressure in the target chamber during the pulse and assuming that the particles in the plasma are pumped away by the target chamber pump, while the neutrals from the plasma source are removed by the source chamber pump. The skimmer plate between the chambers limits the flow of neutrals between the chambers. During this plasma pulse the pressure in the target chamber was $0.8 \mathrm{~Pa}$. With an effective pump speed of $20.000 \mathrm{~m}^{3} / \mathrm{h}, 4.4 \mathrm{~Pa} \mathrm{~m}^{3} / \mathrm{s} \mathrm{H}_{2}$ gas is removed by the target chamber pump. For the plasma beam, this means $2.4 \times 10^{21} \mathrm{H}^{+}$ions per second in total. Assuming again that half of the particle flux is contained by the FWHM area, then the average flux density in this area is $\sim 2 \times 10^{24} \mathrm{~m}^{-2} \mathrm{~s}^{-1}$.

\section{Diagnostic tools}

\subsection{Machine diagnostics}

The basic machine diagnostics such as gas flows, pressures and source voltages are recorded by the CODAC system (via PLCs) and stored in the database automatically during operation. A total of $\sim 150$ of these database variables are currently in use. Another $\sim 100$ variables are in use to describe the experiment (mainly properties of plasma source and the targets used) which are defined before the experiment. During the experiment, a logbook application enables storing log messages and experiment information in the same database. 


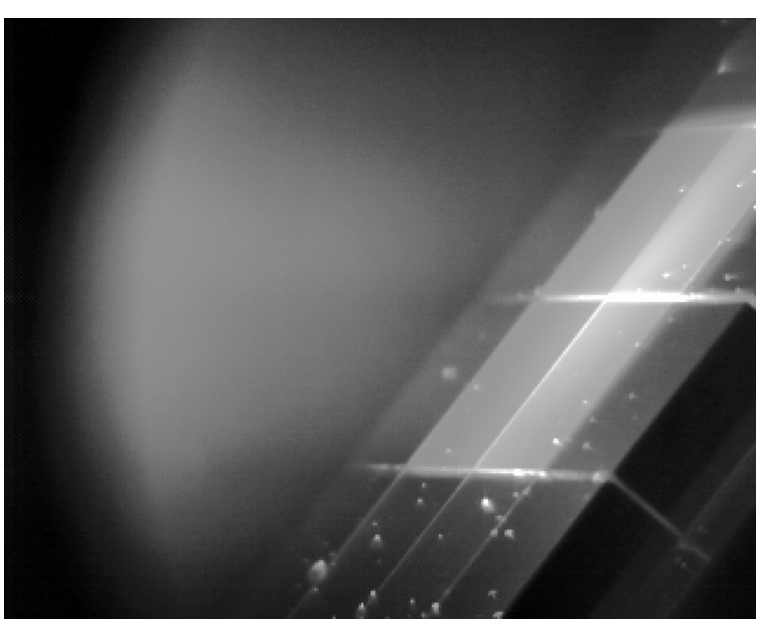

Fig. 5. Infrared image of a tilted castellated target during plasma exposure. On the right, the 'mono-blocks', mounted on a flat target holder, are visible. Temperature of the center block was $\sim 150^{\circ} \mathrm{C}$ during the plasma exposure. The intense light between the blocks is probably caused by a higher emissivity and does not represent a higher temperature.

\subsection{Plasma diagnostics}

Thomson scattering measurements can be done up to $1 \mathrm{~cm}$ in front of the target. The detection system enables to measure 50 spatial channels of $2 \mathrm{~mm}$ each. By averaging 30 laser pulses $(0.6 \mathrm{~J}, 10 \mathrm{~Hz})$ an observational error of $3 \%$ in $n_{\mathrm{e}}$ and $6 \%$ in $T_{\mathrm{e}}$ (at $n_{\mathrm{e}}=1 \times 10^{19} \mathrm{~m}^{-3}$ ) can be obtained, with minimum measurable temperature $T_{\mathrm{e}}<0.1 \mathrm{eV}$.

Calorimetry of several components (e.g.: target and source) is available to determine to power load form the plasma.

Two optical emission (Avantes) spectrometer units (a 2-channel unit: $299-579 \mathrm{~nm}$ and a 4-channel unit: $378-950 \mathrm{~nm}$ ) are used to monitor the plasma light. Impurities such as copper, calcium and oxygen from a failing plasma source can be detected. Additionally, the spectrometers have been used to detect material desorbed during plasma exposure from targets with lithium pre-deposited on them.

A fast visible light (Phantom) camera is available for monitoring short lived events or fluctuations. The camera can record at 6000 frames per second at a $1280 \times 800$ pixels resolution even and higher speeds at lower resolutions.

\subsection{Surface diagnostics}

Target surface temperature measurements during exposure are done with thermal imaging (Fig. 5) and a pyrometer for calibration.
The infrared camera (FLIR SC7500MB) can measure up to $380 \mathrm{~Hz}$ at full frame $(320 \times 256$ pixels $)$ and is calibrated up to $3000^{\circ} \mathrm{C}$.

Laser induced desorption (LID) measurements can be used on targets with plasma implanted molecules such as deuterium. For this technique, a high power (LASAG) laser (60J, 0.1-20 ms, $1064 \mathrm{~nm}$ ) is used in combination with a quadrupole mass spectrometer to determine the desorbed molecular mass and quantity. Additionally, the thermal imaging camera is used to verify the laser spot size and duration. The laser can also be used on the target during plasma exposures to create an additional heat load to simulate ELM-like events [4].

For laser induced breakdown spectroscopy (LIBS) first tests have been performed and the laser beam line has been prepared.

Thermal desorption spectroscopy (TDS) and X-ray photoelectron spectroscopy (XPS) are available at the institute, outside of the Magnum-PSI vacuum system.

\section{Summary}

With the installation of a temporary pulsed magnet and with the availability of reliable and clean plasma sources, the experimental program on Magnum-PSI has started. Continuous (non-pulsed) plasma exposures with a magnetic field up to $2.5 \mathrm{~T}$ (requirement reduced from $3.0 \mathrm{~T}$ ) will be possible when the superconducting magnet is delivered (currently planned for end 2013). The use of an alternative superconducting $1.5 \mathrm{~T}$ magnet with a smaller bore is being prepared in case the $2.5 \mathrm{~T}$ magnet is further delayed.

\section{Acknowledgements}

This work, supported by the European Communities under the contract of Association between EURATOM/FOM, was carried out within the framework of the European Fusion Programme with financial support from NWO. The views and opinions expressed herein do not necessarily reflect those of the European Commission.

\section{References}

[1] J. Rapp, W.R. Koppers, H.J.N. van Eck, G.J. van Rooij, W.J. Goedheer, B. de Groot, et al., Construction of the plasma-wall experiment MagnumPSI, Fusion Engineering and Design 85: (2010) 1455-1459, http://dx.doi.org/ 10.1016/j.fusengdes.2010.04.009.

[2] M.A. van den Berg, S. Brons, O.G. Kruijt, J. Scholten, R. Pasqueta, P.H.M. Smeets, et al., The target for the new plasma/wall experiment Magnum-PSI, Fusion Engineering and Design 86 (2011) 1745.

[3] W.A.J. Vijvers, D.C. Schram, A.E. Shumack, N.J.L. Cardozo, J. Rapp, G.J. van Rooij, Experimental and theoretical determination of the efficiency of a subatmospheric flowing high power cascaded arc hydrogen plasma source, Plasma Sources Science and Technology 19 (2010) 065016.

[4] G. De Temmerman, M.A. van den Berg, T.W. Morgan, T.M. de Kruijf, H.J. van der Meiden, H.J.N. van Eck, et al., High heat flux capabilities of the Magnum-PSI linear plasma device, Fusion Engineering and Design 88 (2013) 483-487.

[5] P.W.C. Groen, V. van Beveren, A. Broekema, P.J. Busch, J.W. Genuit, G. Kaas, et al., Software architecture for control and data acquisition of linear plasma generator Magnum-PSI, Fusion Engineering and Design 88 (2013) 1174-1177. 